\title{
Unappreciated aspects of fluid and electrolyte physiology and implications to patient recovery
}

\author{
Peter Wilkes, MD, PhD • Ayub Akbari, MD
}

Published online: 17 April 2010

(C) Canadian Anesthesiologists' Society 2010

Fluid and electrolyte management of the critically ill patient is a "core skill" for the anesthesiologist and intensivist. Critically ill patients often present with any of a number of insults that result in fluid and electrolyte derangements, and an important part of resuscitative therapy is aggressive fluid and electrolyte management. Once the initiating insult has been stabilized, electrolyte and fluid management often continues to occupy a central component of therapy, and is usually directed at trying to reestablish physiologically appropriate interstitial and vascular compartment volumes. Despite 30 years of research, basic questions are still debated concerning the type of fluid to administer (blood products, artificial colloids, or crystalloids), the amount of fluid to administer (the choice and measurement of appropriate physiological indices), and the clinical scenario wherein fluids should be administered (acute trauma, head injury, sepsis, major surgery, or heart- renal- or hepatic failure, etc.). ${ }^{1}$ Indeed, the challenge of fluid and electrolyte management in the critically ill patient is somewhat analogous to the question of whether a regional or general anesthetic is best for hip replacement in a frail 92-yr-old woman with poor heart function and tenuous kidneys. While there are many components to the question, the answer is often reduced to "a good anesthetic

P. Wilkes, MD, PhD ( ()

Division of Cardiac Anesthesia, University of Ottawa Heart Institute, 40 Ruskin Street, Ottawa, ON K1Y 4W7, Canada

e-mail: pwilkes@ottawaheart.ca

A. Akbari, MD

The Department of Medicine, Division of Nephrology, University of Ottawa, Riverside Campus,

1967 Riverside Drive, Ottawa, ON K1H 7W9, Canada is better than a bad one". By extension, proper fluid and electrolyte management is better than inappropriate management. While the answer may be unsatisfying to residents, they can be re-assured that the answer(s) becomes clearer after they gain a thorough understanding of the physiology of fluid and electrolytes and once they acquire several years of experience.

In their paper in the current issue of the Journal, Stelfox et $a l^{2}$ retrospectively examine the incidence of intensive care unit (ICU) acquired disturbances in plasma sodium concentration $\left[\mathrm{Na}^{+}\right]$in a large population of post cardiac surgical patients. The authors found that the incidence of ICU acquired hypo- and hypernatremia was $12 \%$ and $4 \%$, respectively. More importantly, they demonstrated that hospital mortality in patients with acquired disturbances in $\left[\mathrm{Na}^{+}\right]$, whether hypo- or hypernatremia, was significantly higher than hospital mortality in patients without acquired disturbances in $\left[\mathrm{Na}^{+}\right]$. While the authors correctly pointed out that their study was limited by its retrospective nature, the fact that association was identified rather than causality, and the association was restricted to a large unselected population of patients undergoing heart surgery, the study begs the question - What is the nature of the link between changes in $\left[\mathrm{Na}^{+}\right]$acquired after ICU admission and increased hospital mortality? The answer cannot be unravelled easily because: 1) apart from acute severe hyperkalemia, severe hyponatremia, fulminant pulmonary or cerebral edema, alterations in electrolyte concentrations and/or volume status are not lethal in themselves; and 2) changes in $\left[\mathrm{Na}^{+}\right]$and volume compartments have ramifications throughout the physiological arena.

The answer as to why acutely ill patients develop changes in fluid and electrolyte status is partly a function of: 1) the effects of the initial insult on control mechanisms; 2) the unavoidable consequences of therapy in 
patients whose normal regulatory abilities are attenuated; and 3) perhaps a certain lack of appreciation of the complexity of the physiology of sodium and compartment regulation. A case in point is the use of normal saline as a maintenance solution in the stable yet critically ill patient. It is common knowledge that it is lethal to drink sea water because it is too salty and results in hypernatremia, hyperosmolality, dehydration, and, ultimately, renal failure and death. ${ }^{3}$ However, most residents are not aware of the quantifiable meaning of "too salty", and most are not cognizant of the most concentrated saline solution (fresh water/sea water) that can be provided safely as a maintenance solution. It often comes as a surprise for residents to learn that this concentration is $0.9 \%$ normal saline. Hypertonic saline (3\% salinity) can be tolerated in only relatively small volumes, and, in theory, normal saline can be provided as a maintenance solution, but only if the kidneys can maximally concentrate urine to $>1,000$ $\mathrm{mOsm} \cdot \mathrm{kg}^{-1}$. Since the ability to concentrate urine and the robustness of fluid/electrolyte physiology decreases with age $^{4}$ and concomitant renal disease, it is hardly surprising that using $0.9 \%$ normal saline as a "maintenance solution" can lead to hypernatremia, hyperchloremic acidosis, ${ }^{5,6}$ and hyperosmolality with reduced urine output secondary to increased secretion of antidiuretic hormone. ${ }^{7}$ Normal saline should be reserved for vascular volume replacement or expansion. A healthy person loses water at a greater rate than salt loss through respiratory, cutaneous, gastrointestinal, and urinary routes. Therefore, maintenance solutions require significantly more free water, e.g., $0.45 \%$ saline at 1-1.5 L $\cdot$ day $^{-1}$ for an adult.

A brief review of the underlying physics of compartment physiology illustrates the complexity of salt and volume regulation. The simplest two-compartment system is exemplified by the Donnan equilibrium established by the movement of water and salts between two compartments separated by a semi-permeable membrane, and one of the compartments contains a non-transfusable charged colloid (as in an erythrocyte). Electrochemical gradients described by the Nernst equation determine the distribution of a given electrolyte on either side of the membrane. While correct, the Nernst equation is only a partial description of this system. Unpublished lecture notes by Stewart (described in Wilkes), ${ }^{8}$ demonstrate that a complete description of electrolyte distribution in a simple Donnan system requires the simultaneous solution of 11 equations with three independent variables. The physics becomes more complicated when the system is defined by a capillary bed. As is well known, the Starling equation describes the interplay between hydrostatic and oncotic pressures. While the equation itself would seem straightforward, there is an underlying layer of complexity that is not inherently obvious. As reviewed by Lucas, ${ }^{9}$ Starling predicted over a century ago that a change in one factor in the equation would affect all other factors, thus confirming the Niels Bohr and Yogi Berra truism that "predictions are very difficult, especially about the future." Indeed, a complete description of the system pertaining to a capillary bed is reportedly described by the simultaneous solution of 26 equations incorporating ten independent variables (Stewart, unpublished lecture notes). Understanding normal physiology is not easy; thus, it is not surprising that treatment of pathophysiology is even more challenging.

While a degree of volume overload is often tolerated as a consequence of the initial resuscitative efforts, it should not be viewed as benign. ${ }^{10-12}$ If volume overload cannot be prevented, it must eventually be corrected, usually with diuretic and sometimes with renal replacement therapy. While this approach is certainly appropriate, the role of muscle movement and the resulting improvement in lymphatic flow is central to fluid mobilization and re-establishment of homeostasis. ${ }^{13}$ In their recent randomized control trial, Schweickert et al. ${ }^{14}$ found that physical and occupational therapy in the earliest stages of critical illness resulted in better functional outcomes at hospital discharge, shorter duration of delirium, and more ventilator-free days.

As suggested above, the functional significance attributable to the degree to which the components of physiology are non-linearly interconnected is often not fully appreciated. Inter-connectivity and non-linearity are at the core of biological variability and chaos theory, and, although they are well beyond the scope of this editorial, they are likely to be of profound clinical relevance. ${ }^{15}$ A simple example of interconnectivity between systems is the dependence of acid-base status on electrolytes. This was appreciated by Gamble in the 1930s, ${ }^{\mathrm{A}, \mathrm{B}}$ and it was quantitatively formalized by Peter Stewart over 30 years ago. ${ }^{16}$ The consequence of non-linear system interconnectivity to patient care in the clinical setting is that it will likely not be possible to ascertain causality between fluid and electrolyte management and increased mortality. Disturbances in $\left[\mathrm{Na}^{+}\right]$do not occur in isolation. A change in sodium content within the extracellular compartment will alter water content and hence compartment volume, with consequences to cardiovascular hemodynamics and renal function. Movement of $\left[\mathrm{Na}^{+}\right]$will directly alter hydrogen $\left[\mathrm{H}^{+}\right]$through change in the strong ion difference. Perturbations in $\left[\mathrm{H}^{+}\right]$have ramifications throughout physiology. ${ }^{17}$

\footnotetext{
${ }^{\text {A }}$ Gamble JL. Extracellular fluid: extracellular fluid and its vicissitudes. Bulletin of the Johns Hopkins Hospital 1937; LXI: 151-73.

B Gamble JL. Extracellular fluid: renal defense of extracellular fluid: control of acid-base excretion and the factors of water expenditure. Bulletin of the Johns Hopkins Hospital 1937; LXI: 174-97.
} 
It is probable that the linkage between hospital mortality and acquired disturbances in volume and electrolyte status will remain undefined for the foreseeable future of critical care medicine. Conceptually, change in fluid/electrolyte homeostasis may represent 1) primary pathophysiology, 2) iatrogenic events, or 3) a physiologically appropriate response to the altered physiological state of the long-term intensive care patient. ${ }^{8}$ These options are not mutually exclusive. While direct causality may never be established and, indeed, may not exist, it would seem prudent to take a similar approach to fluid and electrolyte management as exists for glycemic control. Whether colloids or crystalloids are used, they should be used with as complete an understanding of physiological processes and patient specific endpoints as possible. In the paper by Stelfox et al., ${ }^{2}$ a major finding was the increased mortality that resulted from a small modification in sodium concentration (a median of $2 \mathrm{mmol} \cdot \mathrm{L}^{-1} \cdot \mathrm{day}^{-1}$ for hyponatremia and a median of $1.5 \mathrm{mmol} \cdot \mathrm{L}^{-1} \cdot \mathrm{day}^{-1}$ for hypernatremia), a change that most clinicians would consider inconsequential. It would seem unlikely that maintaining $\left[\mathrm{Na}^{+}\right]$, and indeed all electrolytes, within their normal range would be harmful.

\section{Les aspects méconnus de la physiologie des liquides et des électrolytes et ses implications pour la récupération du patient}

La prise en charge liquidienne et électrolytique des patients gravement malades est une «compétence de base » de l'anesthésiologiste et de l'intensiviste. Les patients gravement malades manifestent souvent toutes sortes de lésions qui sont le résultat de déséquilibres aux niveaux liquidien et électrolytique, et une prise en charge dynamique des liquides et des électrolytes constitue une part importante du traitement de réanimation. Une fois la lésion d'origine stabilisée, la prise en charge électrolytique et liquidienne continue souvent d'occuper une place prépondérante dans le traitement. Cette prise en charge vise généralement à rétablir les volumes des compartiments interstitiel et vasculaire de façon adaptée d'un point de vue physiologique. Malgré trente ans de recherches, certaines questions de base font toujours l'objet de débats, notamment le type de liquide à administrer (produits sanguins, colloïdes artificiels, ou cristalloïdes), la quantité de liquides à administrer (la sélection et la mesure d'indices physiologiques adéquats) et les cas cliniques dans lesquels des liquides devraient être administrés (traumatisme aigu, traumatisme crânien, sepsie, chirurgie majeure, ou insuffisance cardiaque/rénale ou hépatique, etc.). ${ }^{1}$ En effet, le défi que représente la prise en charge liquidienne et électrolytique du patient gravement malade est similaire à la question de savoir s'il est mieux d'utiliser un anesthésique régional ou général pour une chirurgie de remplacement de la hanche chez une femme de 92 ans de santé fragile avec une mauvaise fonction cardiaque et des reins fragilisés. Bien que la question comporte de nombreuses dimensions, la réponse se résume souvent à « un bon anesthésique vaut mieux qu'un mauvais ». Ainsi, une prise en charge liquidienne et électrolytique adaptée sera préférable à une mauvaise prise en charge. Bien que la réponse puisse paraître insuffisante pour les résidents, il est possible de les rassurer en leur expliquant que la ou les réponses deviennent plus claires une fois qu'ils ont acquis une compréhension plus globale de la physiologie des liquides et des électrolytes, et après plusieurs années d'expérience.

Dans leur article dans ce numéro du Journal, Stelfox et coll. $^{2}$ ont étudié rétrospectivement l'incidence de perturbations de la concentration plasmatique de sodium $(\mathrm{Na}+)$ acquises à l'unité des soins intensifs (USI) chez une vaste population de patients ayant subi une chirurgie cardiaque. Les auteurs ont noté que l'incidence d'hypo- et d'hypernatrémie acquises à l'USI était de $12 \%$ et $4 \%$, respectivement. Qui plus est, ils ont démontré que la mortalité hospitalière des patients présentant des déséquilibres du $\mathrm{Na}+$, qu'il s'agisse d'hypo- ou d'hypernatrémie, était significativement plus élevée que la mortalité hospitalière des patients n'ayant pas manifesté de déséquilibre du $\mathrm{Na}+$. Bien que les auteurs aient souligné le fait que leur étude était limitée de par sa nature rétrospective, il n'en demeure pas moins qu'une association, plutôt qu'une relation de causalité, a été remarquée. En outre, cette association s'est limitée à une vaste population non choisie de patients subissant une chirurgie cardiaque. Dès lors, cette étude soulève la question suivante : De quelle nature est le lien entre les changements de $\mathrm{Na}+$ acquis après l'admission à l'USI et l'augmentation de la mortalité hospitalière? Il n'y a pas de réponse simple à cette question, parce que : 1) hormis une hyperkaliémie aiguë grave, l'hyponatrémie grave, les œdèmes pulmonaires ou cérébraux fulminants et les changements dans les concentrations électrolytiques et/ou l'état de volume ne sont pas fatals en soi; et 2) les changements du $\mathrm{Na}+$ et des compartiments de volume ont des répercussions sur toute la physiologie.

La réponse à la question de savoir pourquoi des patients gravement malades manifestent des changements de leur état liquidien et électrolytique est, en partie, fonction : 1) des effets de la lésion initiale sur les mécanismes de contrôle, 2) des conséquences inévitables d'un traitement chez des patients dont les capacités régulatrices normales sont mitigées; et 3) peut-être d'un certain manque de reconnaissance de la complexité de la physiologie sodique et de la régulation des compartiments. Un exemple typique est 
l'utilisation de sérum physiologique comme solution de maintien chez le patient stable mais gravement malade. Il est bien connu qu'il est fatal de boire de l'eau de mer parce qu'elle est trop salée, ce qui peut provoquer de l'hypernatrémie, de l'hyperosmolalité, de la déshydratation et, en fin de compte, une insuffisance rénale et la mort. ${ }^{3}$ Pourtant, la plupart des résidents ne sont pas conscients de la signification quantifiable de ce qu'on entend par « trop salé », et la plupart ne savent pas quelle est la concentration maximale d'une solution salée (eau douce/eau de mer) qui puisse être administrée de façon sécuritaire comme solution de maintien. Les résidents sont souvent surpris lorsqu'ils apprennent qu'il s'agit de sérum physiologique à $0,9 \%$. Une solution saline hypertonique ( $3 \%$ de salinité) peut être tolérée seulement en volume relativement restreint et, en théorie, du sérum physiologique peut être administré comme solution de maintien, mais seulement si les reins peuvent concentrer l'urine de façon maximale à $>1$ $000 \mathrm{mOsm} \cdot \mathrm{kg}^{-1}$. Étant donné que la capacité de concentrer de l'urine et la robustesse de la physiologie liquidienne/ électrolytique diminue avec l'âge ${ }^{4}$ et les maladies rénales concomitantes, il n'est dès lors pas surprenant que l'utilisation d'une solution saline à $0,9 \%$ comme « solution de maintien » puisse provoquer de l'hypernatrémie, de l'acidose hyperchlorémique ${ }^{5,6}$ et de l'hyperosmolalité en raison d'une production réduite d'urine secondaire à une sécrétion accrue de l'hormone antidiurétique. ${ }^{7}$ Il faudrait réserver le sérum physiologique pour le remplacement ou l'expansion du volume vasculaire. Une personne en bonne santé perd de l'eau plus rapidement que du sel par les voies respiratoires, cutanées, gastro-intestinales et urinaires. C'est pourquoi les solutions de maintien nécessitent significativement plus d'eau, par exemple une solution saline à $0,45 \%$ à 1-1,5 L jour $^{-1}$ chez un adulte.

Un examen de la physique sous-jacente à la physiologie de compartimentalisation illustre la complexité de la régulation du sel et du volume. Le système à deux compartiments le plus simple est exemplifié par l'équilibre de Donnan. Ce dernier s'établit par le mouvement de l'eau et des sels entre deux compartiments séparés par une membrane semi-perméable, où l'un des compartiments contient un colloïde chargé qui ne peut être transfusé (comme dans un érythrocyte). Les gradients électrochimiques décrits par l'équation de Nernst déterminent la distribution d'un électrolyte donné de part et d'autre de la membrane. Bien qu'elle soit correcte, l'équation de Nernst ne constitue qu'une description partielle de ce système. Des notes de conférence non publiées de Stewart (décrites par Wilkes) ${ }^{8}$ démontrent qu'une description complète de la distribution électrolytique dans un simple système de Donnan nécessite la solution simultanée de 11 équations avec trois variables indépendantes. La physique se complique lorsque le système est défini par un lit capillaire. Il est bien connu que l'équation de Starling décrit l'interaction entre les pressions hydrostatiques et oncotiques. Alors que l'équation en soi paraît simple, elle comprend une couche sous-jacente de complexité qui n'est pas évidente de prime abord. Comme Lucas l'a remarqué, ${ }^{9}$ Starling prédisait il y a plus d'un siècle qu'un changement de l'un des facteurs de l'équation affecterait tous les autres facteurs, confirmant ainsi le truisme de Niels Bohr et Yogi Berra selon lequel « les prédictions sont très difficiles, particulièrement celles qui touchent à l'avenir ». En effet, une description complète du système de Donnan portant sur un lit capillaire est décrite par la solution simultanée de 26 équations intégrant dix variables indépendantes (Stewart, notes de conférence non publiées). La compréhension d'une physiologie normale n'est pas aisée; dès lors, il n'est pas surprenant que le traitement d'une physiopathologie soit encore plus difficile.

Alors qu'un certain niveau de surcharge volumique est souvent toléré à la suite des efforts initiaux de réanimation, il ne faut pas considérer cette situation comme bénigne. ${ }^{10-12} \mathrm{Si}$ la surcharge volumique ne peut être évitée, elle doit être ultimement corrigée, en général à l'aide d'un traitement diurétique et parfois avec une thérapie de remplacement rénal. Bien que cette approche soit sans aucun doute adaptée, le rôle des mouvements musculaires et l'amélioration du flux lymphatique qui en résulte est essentielle à la mobilisation des liquides et à la restauration de l'homéostase. ${ }^{13}$ Dans leur étude randomisée avec placebo récente, Schweickert $e t$ coll. ${ }^{14}$ ont découvert que la physiothérapie et l'ergothérapie réalisées aux stades les plus précoces d'une maladie grave avaient pour résultats de meilleurs devenirs fonctionnels lors du congé de l'hôpital, une durée réduite du delirium et davantage de journées sans ventilateur.

Comme nous l'avons suggéré plus haut, l'importance fonctionnelle qu'il faut attribuer au niveau d'interconnectivité non linéaire des différentes composantes de la physiologie est souvent méconnue. L'interconnectivité et la non-linéarité sont au centre de la variabilité biologique et de la théorie du chaos et, quoique ces deux phénomènes soient bien au-delà de la portée de cet éditorial, il est probable qu'ils aient une pertinence clinique prépondérante. ${ }^{15} \mathrm{Un}$ exemple simple d'interconnectivité entre systèmes est la dépendance de l'état acido-basique sur les électrolytes. Ce phénomène a été décrit par Gamble dans les années 1930,, et il a été formalisé de façon quantitative par Peter Stewart il y a plus de 30 ans. ${ }^{16}$ La conséquence d'une interconnectivité non linéaire sur les soins aux patients dans un contexte

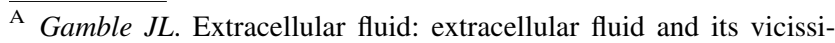
tudes. Bulletin of the Johns Hopkins Hospital 1937; LXI: 151-73.

B Gamble JL. Extracellular fluid: renal defense of extracellular fluid: control of acid-base excretion and the factors of water expenditure. Bulletin of the Johns Hopkins Hospital 1937; LXI: 174-97.
} 
clinique est qu'il sera vraisemblablement impossible d'établir un lien de causalité entre la prise en charge liquidienne et électrolytique et une augmentation de la mortalité. Les dérèglements au niveau du $\mathrm{Na}+$ ne surviennent pas de façon isolée. Un changement au niveau du contenu sodique dans le compartiment extracellulaire modifiera le contenu aqueux et, par conséquent, le volume du compartiment, ce qui aura alors des répercussions sur l'hémodynamie cardiovasculaire et la fonction rénale. Les modifications sodiques provoqueront des changements directs de l'hydrogène $(\mathrm{H}+)$ en raison des changements dans la différence ionique élevée. Les perturbations au niveau du $\mathrm{H}+$ ont des ramifications dans toute la physiologie. ${ }^{17}$

Il est probable que le lien entre la mortalité à l'hôpital et les dérèglements acquis au niveau du volume et de l'état électrolytique demeurera indéterminé dans les années à venir de la médecine des soins critiques. D'un point de vue conceptuel, des changements de l'homéostasie liquidienne/électrolytique pourraient représenter : 1) une physiopathologie primaire; 2) des complications iatrogéniques; ou 3) une réponse physiologique adaptée à l'état physiologique altéré du patient aux soins intensifs à long terme. ${ }^{8}$ Ces différentes possibilités ne sont pas mutuellement exclusives. Alors qu'il est possible qu'une causalité directe ne soit jamais établie—voire n'existe pas-, il semble prudent d'adopter une approche de la prise en charge liquidienne et électrolytique semblable à celle qui existe pour le contrôle du glucose. Qu'il s'agisse de colloïdes ou de cristalloïdes, les liquides devraient être utilisés avec une compréhension aussi complète que possible des processus physiologiques et des résultats pertinents au patient. Dans l'article de Stelfox et coll., ${ }^{2}$ une des découvertes principales est l'augmentation de la mortalité résultant d'une légère modification de la concentration sodique (une médiane de $2 \mathrm{mmol} \cdot \mathrm{L}^{-1} \cdot$ jour $^{-1}$ pour l'hyponatrémie et une médiane de $1,5 \mathrm{mmol} \cdot \mathrm{L}^{-1} \cdot \mathrm{jour}^{-1}$ pour l'hypernatrémie), un changement que la plupart des cliniciens considéreraient comme sans conséquence. Il semblerait improbable que le maintien du $\mathrm{Na}+$, et de tous les électrolytes, à l'intérieur de leur plage de valeurs normales, soit néfaste.

Competing interests None declared.

\section{References}

1. Holte K, Sharrock NE, Kehlet H. Pathophysiology and clinical implications of perioperative fluid excess. Br J Anaesth 2002; 89: 622-32.

2. Stelfox HT, Ahmed SB, Zygun D, Khandwala F, Laupland K. Characterization of intensive care unit acquired hyponatremia and hypernatremia following cardiac surgery. Can J Anesth 2010; 57. doi:10.1007/s12630-010-9309-1.

3. Etzion Z, Yagil R. Metabolic effects in rats drinking increasing concentrations of sea-water. Comp Biochem Physiol A Comp Physiol 1987; 86: 49-55.

4. Andreucci VE, Russo D, Cianciaruso B, Andreucci M. Some sodium, potassium and water changes in the elderly and their treatment. Nephrol Dial Transplant 1996; 11(Suppl 9): 9-17.

5. Scheingraber S, Rehm M, Sehmisch C, Finsterer U. Rapid saline infusion produces hyperchloremic acidosis in patients undergoing gynecologic surgery. Anesthesiology 1999; 90: 1265-70.

6. Rehm M, Orth V, Scheingraber S, Kreimeier U, Brechtelsbauer $H$, Finsterer $U$. Acid-base changes caused by $5 \%$ albumin versus $6 \%$ hydroxyethyl starch solution in patients undergoing acute normovolemic hemodilution: a randomized prospective study. Anesthesiology 2000; 93: 1174-83.

7. Hall J, Robertson G. Diabetes insipidus. In: Kirby RR, Taylor RW. Problems in Critical Care, vol. 4, Endocrine Emergencies. Philadelphia: JB Lippincott; 1990: 343.

8. Wilkes P. Normal [SID]. In: Kellum JA, Elbers PW. Stewart's Textbook of Acid-Base. Amsterdam: Acidbase.org; 2009: 201-16.

9. Lucas CE. The water of life: a century of confusion. J Am Coll Surg 2001; 192: 86-93.

10. Lowell JA, Schifferdecker C, Driscoll DF, Benotti PN, Bistrian $B R$. Postoperative fluid overload: not a benign problem. Crit Care Med 1990; 18: 728-33.

11. Weinstein PD, Doerfler ME. Systemic complications of fluid resuscitation. Crit Care Clin 1992; 8: 439-48.

12. Bagshaw SM, Bellomo R. The influence of volume management on outcome. Curr Opin Crit Care 2007; 13: 541-8.

13. Koomans HA, Boer WH. Causes of edema in the intensive care unit. Kidney Int Suppl 1997; 59: S105-10.

14. Schweickert WD, Pohlman MC, Pohlman AS, et al. Early physical and occupational therapy in mechanically ventilated, critically ill patients: a randomised controlled trial. Lancet 2009; 373: 1874-82.

15. Seely AJ, Christou NV. Multiple organ dysfunction syndrome: exploring the paradigm of complex nonlinear systems. Crit Care Med 2000; 28: 2193-200.

16. Stewart $P A$. Independent and dependent variables of acid-base control. Respir Physiol 1978; 33: 9-26.

17. Gauthier PM, Szerlip HM. Metabolic acidosis in the intensive care unit. Crit Care Clin 2002; 18: 289-308, vi. 